\title{
Performance of Point-of-Care Testing Compared with the Standard Laboratory Diagnostic Test in the Measurement of HbA1c in Indonesian Diabetic and Nondiabetic Subjects
}

\author{
Afiat Berbudi $\mathbb{D}^{1,2}$ Nofri Rahmadika, ${ }^{2}$ Adi Imam Tjahjadi, ${ }^{2,3}$ and Rovina Ruslami ${ }^{2,4}$ \\ ${ }^{1}$ Department of Biomedical Sciences, Parasitology Division, Faculty of Medicine, Universitas Padjadjaran, Bandung, Indonesia \\ ${ }^{2}$ Infectious Disease Research Centre, Faculty of Medicine, Universitas Padjadjaran, Bandung, Indonesia \\ ${ }^{3}$ Department of Biomedical Sciences, Microbiology Division, Faculty of Medicine, Universitas Padjadjaran, Bandung, Indonesia \\ ${ }^{4}$ Department of Biomedical Sciences, Pharmacology and Therapy Division, Faculty of Medicine, Universitas Padjadjaran, \\ Bandung, Indonesia
}

Correspondence should be addressed to Afiat Berbudi; a.berbudi@unpad.ac.id

Received 28 February 2020; Revised 29 May 2020; Accepted 19 June 2020; Published 11 July 2020

Academic Editor: Hiroshi Okamoto

Copyright (C) 2020 Afiat Berbudi et al. This is an open access article distributed under the Creative Commons Attribution License, which permits unrestricted use, distribution, and reproduction in any medium, provided the original work is properly cited.

\begin{abstract}
Objective. This study is aimed at investigating if point-of-care testing for HbAlc (POCT-HbAlc) using the HemoCue ${ }^{\circledR} \mathrm{HbA1c} 501$ system could be an alternative method for diabetes screening and monitoring to replace the HbAlc measurement in a standard diagnostic laboratory. Design. This was a cross-sectional study to assess the agreement between POCT and a standard laboratory measurement method for determining the level of HbAlc. Setting and Participants. In total, 108 participants were recruited to participate in this study, consisting of 61 diabetics and 47 nondiabetics. The diabetic group comprised 37 females and 24 males, diagnosed with type 2 diabetes mellitus (DM) and undergoing diabetes treatment at several community health care centres in Bandung, West Java. The nondiabetic group consisted of 15 female and 32 male patients of several community health care centres and healthy volunteers. Sample Collection and Analysis. A venous blood sample was taken for routine HbA1c analysis by the diagnostic laboratory method. For the POCT-HbAlc, a blood sample was taken from the fingertip at the same time and analysed with the HemoCue ${ }^{\circledR}$ HbAlc 501 system. Outcome Measures. The HbA1c results of both methods were compared and analysed with a Bland-Altman agreement plot. The sensitivity and specificity of the POCT-HbA1c data were also compared with those of the standard diagnostic results. Results. Based on the Bland-Altman plot, the HbA1c level for 100 out of 108 $(92.59 \%)$ subjects analysed by the POCT-HbAlc was within the range of the $95 \%$ limit of agreement. Compared with the standard diagnostic assay, the sensitivity of the POCT-HbA1c was $97.83 \%$ and its specificity was $77.42 \%$. Conclusions. The high sensitivity and accuracy of POCT-HbAlc indicate that it is a potential method for diabetes screening and monitoring to replace the routine diagnostic laboratory $\mathrm{HbAlc}$ measurement, especially when a rapid result is required.
\end{abstract}

\section{Introduction}

Diabetes is a global health problem, indicated by a high blood glucose level caused by insufficient insulin production by the pancreas or decreasing insulin sensitivity [1]. Uncontrolled blood glucose in diabetes is associated with an increased risk of several complications, such as organ damage, cancer, dementia, and susceptibility to infection [2-8].

Early diagnosis of diabetes and associated complications provides an opportunity to commence timely effective pre- ventive treatment that reduces the subsequent development or progression of macrovascular and microvascular disease. The key to preventing diabetic complications is by maintaining the blood glucose level within the normal range [9-11]. Consequently, measurements of fasting and 2-hour postprandial blood glucose are widely used in diabetes diagnosis and management [12]. However, the success of therapy and patient compliance is not well reflected by the blood glucose test at a specific time. Glucose bound with haemoglobin (glycated haemoglobin), known as $\mathrm{HbA1c}$, is commonly used 
to evaluate blood glucose control over the previous 2-3 months $[13,14]$, with HbA1c levels higher than $6.5 \%$ indicating uncontrolled blood glucose level [15].

Information regarding the HbAlc level could help physicians to improve the management of diabetic patients through proper monitoring [16-19]. HbAlc testing has been included for diagnosing diabetes in the American Diabetes Association (ADA) guidelines since 2010 [20], and the World Health Organisation (WHO) also recommends $\mathrm{HbA} 1 \mathrm{c}$ testing for diagnosing diabetes [21]. Similarly, recommendations to use HbAlc testing in diabetes diagnosis have been issued by the United Kingdom and New Zealand $[22,23]$, with the Australian Diabetes Society (ADS) expert committee also recommending $\mathrm{HbAlc}$ assessment to diagnose diabetes and be utilised for a corresponding Medicare Benefits Schedule (MBS) [24, 25]. HbA1c assessment benefits diabetic patients in several ways, including no requirement for fasting, less biological variability, and indicating an increased risk of cardiovascular disease (CVD) and retinopathy, compared to an oral glucose tolerance test (OGTT) or fasting glucose levels $[26,27]$. Even though HbA1c assessment is important and beneficial in diabetes mellitus (DM) patient management, it is still not commonly used in Indonesia $[28,29]$ as it is costly and time-consuming.

Point-of-care testing (POCT) helps to ensure that after the diagnostic test, the patient receives the appropriate treatment in the hospital or the clinic [30]. Immediate results provided by POCT are important for reducing diabetes diagnosis time and initiating treatment for patients in remote areas [31]. Over the past few years, several medical device companies have produced rapid examination instruments for $\mathrm{HbA1c}$, which generate the result in minutes, thus helping health workers, particularly doctors, to make rapid clinical decisions regarding treatment [32]. Nevertheless, the accuracy of the POCT-HbA1c measurement is still questionable $[23,33]$; therefore, this study was conducted to compare the results of the POCT using the HemoCue ${ }^{\circledR} \mathrm{HbAlc}$ 501 system with those of the standard laboratory method to evaluate whether this POCT could replace the standard laboratory methodology in Indonesia.

\section{Materials and Methods}

2.1. Research Subject and Sampling. The analysis was conducted by comparing the HbA1c level of diabetic and nondiabetic subjects obtained by the POCT-HbA1c with that of the standard diagnostic laboratory method. The diabetic group comprised 61 individuals from several community health care centres in Bandung that had been diagnosed with type 2 diabetes and were undergoing diabetes treatment. Nondiabetic subjects did not have diabetes symptoms and normal $\mathrm{HbA1c}$ and blood glucose levels. After giving informed consent, $5 \mathrm{ml}$ of blood was collected to measure the HbAlc by the diagnostic laboratory test "Prodia" that has the National Glyco-Haemoglobin Standardisation Programme (NGSP) accreditation. The HbA1c level was analysed using highperformance liquid chromatography per WHO guidelines [12]. For the POCT-HbA1c, $5 \mu \mathrm{l}$ of blood was sampled from the fingertip at the same time and was analysed using the
HemoCue $^{\circledR}$ HbA1c 501 system (HemoCue AB, Sweden) certified by the International Federation of Clinical Chemistry and Laboratory Medicine (IFCC) and the NGSP according to the manufacturer's protocol. Briefly, the cartridge was inserted into the cartridge compartment. The reagent pack was prepared and applied to the blood specimen, then inserted into the cartridge, with the result automatically displayed after 5 minutes.

2.2. Analysis of Agreement Test. Bland-Altman plot statistical analysis was used to compare the $\mathrm{HbAlc}$ results obtained through the POCT using the HemoCue ${ }^{\circledR}$ HbA1c 501 system with those obtained by the standard diagnostic laboratory method.

2.3. Sensitivity and Specificity of POCT-HbA1c. The sensitivity and specificity of the POCT method using the HemoCue ${ }^{\circledR}$ HbA1c 501 system were compared with those of the standard laboratory method as a reference, using the standard cut-off HbA1c level of $6.5 \%$ [15].

2.4. Ethical Approval. All study participants were provided with the study information and gave written informed consent. All study procedures and methods were approved by the Ethical Committee of the Faculty of Medicine, Universitas Padjadjaran, Indonesia (No. 69/UN6.KEP/EC/2018).

\section{Results}

The mean value of $\mathrm{HbA} 1 \mathrm{c}$ was $6.96 \%$ for the standard laboratory measurement versus $7.15 \%$ for POCT-HbA1c, with a mean difference of -0.187. A comparison of HbA1c measurements by the two methods is presented in Supplementary data 1.

3.1. Accuracy of HbA1c Measurement Using POCT-HbA1c. According to the Bland-Altman plot analysis, the HbAlc levels of 100 out of 108 subjects analysed with POCT$\mathrm{HbAlc}$ and the standard diagnostic laboratory method were within the range of agreement limits (95\% confidence intervals: -1.675 to 1.301 ), with only 3 upper outliers and 5 lower outliers outside the agreement limits range (Figure 1). This indicates that $92.59 \%$ of the HbAlc measurements by the HemoCue HbA1c 501 system are in line with the standard laboratory method.

\subsection{POCT-HbA1c Using the HemoCue HbA1c 501 System Is} Less Costly and More Rapid Compared to the Standard Diagnostic Laboratory HbA1c Method. A comparison of both methods in terms of unit cost, measurement time, required blood volume, and the analysis process is presented in Table 1. The POCT-HbA1c method is less costly, requiring fewer resources to rapidly measure $\mathrm{HbAlc}$ compared with the standard laboratory analysis. Furthermore, it only requires a small blood sample from the patient's fingertips, whereas the laboratory analysis requires a venous blood sample collected by a trained phlebotomist.

3.3. POCT-HbA1c Using the HemoCue ${ }^{\circledR}$ HbAlc 501 Demonstrates High Sensitivity Comparable to That of the Standard Laboratory Method. Based on the current guidelines, 


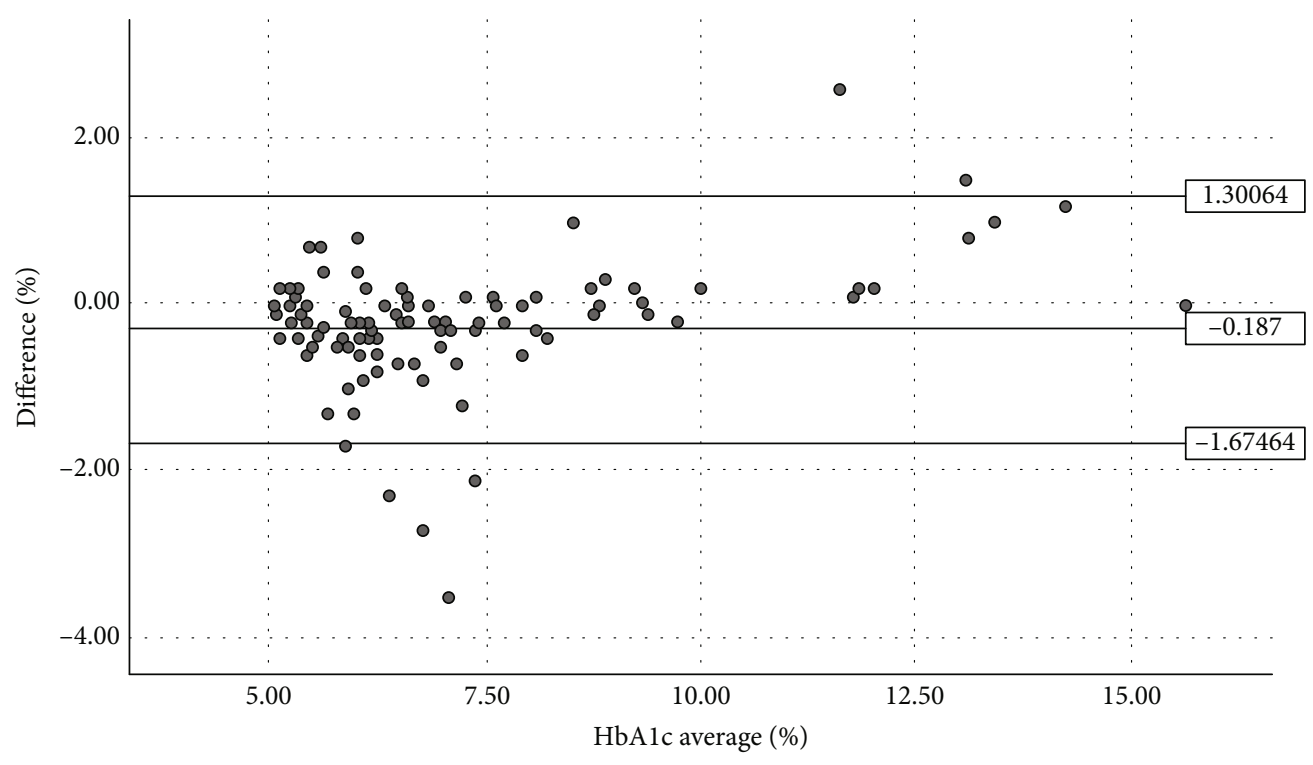

Figure 1: Analysis of the agreement between the POCT-HbA1c using the HemoCue HbA1c 501 system and standard diagnostic laboratory method (SD of bias $=0.759 ; 95 \%$ limits of agreement $=-1.675-1.301 ; n=108$ ).

TABLE 1: A comparison of HbA1c measurement by POCT-HbA1c and the standard laboratory method.

\begin{tabular}{lcc}
\hline Item & $\begin{array}{c}\text { Laboratory } \\
\text { test of HbA1c }\end{array}$ & POCT-HbAlc \\
\hline Unit cost/sample (USD)* & 12.4 & 5.5 \\
Time to get the result & 2 days & 5 minutes \\
Required blood volume & $\sim 1 \mathrm{ml}$ & 1 drop $(\sim 4 \mu \mathrm{l})$ \\
Blood collection method & Phlebotomy & Finger prick \\
Sample processing & Laboratory & Bedside/clinic \\
\hline
\end{tabular}

${ }^{*}$ Unit cost in Indonesia.

intensive therapy will be given to patients with an $\mathrm{HbA1c}$ level of more than $6.5 \%$. To evaluate the clinical use of POCT-HbA1c, the HbA1c results were compared with those of the standard laboratory results using a cut-off point of $6.5 \%$ for $\mathrm{HbA} 1 \mathrm{c}$, as shown in Table 2 . The $2 \times 2$ cross table reveals that the POCT-HbA1c has a sensitivity of $97.83 \%$, while the specificity of this tool was $77.42 \%$. The probability of having diabetes in a subject with $\mathrm{HbA1c} \geq 6.5$ tested by POCT-HbA1c (positive predictive value) was $76.27 \%$, while the probability of not having diabetes in a subject with $\mathrm{HbA} 1 \mathrm{c}<6.5$ tested by POCT-HbAlc (negative predictive value) was $97.96 \%$. The diagnostic accuracy (effectiveness) of POCT-HbAlc expressed as a proportion of true positives and true negatives (correctly classified subjects by POCTHbA1c) in all subjects was $86.11 \%$.

\section{Discussion}

Fasting blood sugar level is the most commonly used indicator of diabetic patients' blood sugar control and compliance with treatment. Despite its effectiveness and low cost, fasting blood sugar level measurement is inconvenient since the
TABLE 2: Sensitivity and specificity of the POCT results using the HemoCue HbA1c 501 system based on a $6.5 \%$ cut-off point.

\begin{tabular}{lcc}
\hline HbAlc test by POCT (\%) & \multicolumn{2}{c}{ HbA1c test by standard } \\
& laboratory method (\%) \\
& HbAlc $\geq 6.5$ & HbA1c $<6.5$ \\
\hline HbA1c $\geq 6.5$ & 45 & 14 \\
HbAlc $<6.5$ & 1 & 48 \\
Total & 46 & 62 \\
\hline
\end{tabular}

${ }^{*}$ The values in the table represent the number of subjects $(n=108)$. Sensitivity (45 out of $46 / 97.83 \%$ ), specificity ( 48 out of $62 / 77.42 \%$ ), positive predictive value ( 45 out of 59/76.27\%), negative predictive value (48 out of $49 / 97.96 \%$ ), false positive (14 of $62 / 22.58 \%$ ), false negative ( 1 of $46 / 2.17 \%$ ), and accuracy $(86.11 \%)$.

patient must have fasted for 8 hours before blood collection. Hence, HbAlc measurement is used to predict the long-term complications of diabetes, with a high HbAlc closely related to an increased risk of CVD, nephropathy, and retinopathy; therefore, it could further predict the risk of mortality for diabetics [34]. The Centers for Disease Control and Prevention states that "every drop of HbAlc in the blood (for example, from $8.0 \%$ to $7.0 \%$ ) could reduce microvascular complication risk to the eye, kidney, and nerve diseases up to $40 \%$. Therefore, the HbAlc level is crucial for appropriate treatment" [32]. Currently, the ADA and the American Association of Clinical Endocrinologists recommend diabetics achieve an HbAlc level of $6.5 \%$ as an indicator of successful blood glucose control $[15,35]$.

POCT-HbAlc gives a more rapid result compared with laboratory-based $\mathrm{HbAlc}$ measurement, and the longer time gives the patient more opportunity to be noncompliant in checking their HbA1c levels [36, 37]. POCT-HbA1c makes it possible to monitor patients' prognosis and adherence to treatment in one visit [32]. Nevertheless, the controversy over the accuracy of POCT is still a concern for the users 
$[38,39]$. Devices have been designed to accurately measure $\mathrm{HbAlc}$, as it has become an important component in diabetes management [15], such as the HemoCue system. Such systems are advantageous, particularly for patients who have difficulties attending the laboratory for blood tests or repeat visits [40]. A study by Cagliero et al. showed that the HbA1c test result during the same visit was associated with increasing glycaemic control in diabetics [17]. It could also be an alternative to help with diagnosis, especially in areas where a laboratory is not available and is financially constrained or there is inadequate transportation to support referring patients to a more complete health infrastructure.

In this study, the mean difference between the POCT$\mathrm{HbAlc}$ measurements and the standard lab was only -0.187 , indicating that the POCT-HbAlc measurements were in line with the $\mathrm{HbAlc}$ measured by the standard diagnostic lab method. One hundred of 108 (92.59\%) of the HbAlc measurements using the HemoCue ${ }^{\circledR} \mathrm{HbA1c} 501$ system were within the $95 \%$ limit of agreement, with only 8 (7.41\%) outliers. The level of agreement between these results indicates that POCT using the HemoCue can be useful for monitoring blood glucose levels, supporting the clinician to manage the patient and improve the outcome of diabetes therapy.

Regarding the sensitivity and specificity, POCT-HbAlc using the HemoCue system was sensitive (92.83\%) but less specific (specificity $77.42 \%$ ) as $22.58 \%$ of nondiabetic subjects were identified as having an $\mathrm{HbA} 1 \mathrm{c} \geq 6.5$. Nonetheless, the proportion of positive results to population number (diagnostic accuracy) was high (86\%).

Early detection of diabetes is important to prevent the disease progression and associated complications including coronary heart disease, stroke, cancer, or damage to various organs such as the kidneys, eyes, and nerves [27, 41]. Since screening is crucial for the early diagnosis of diabetes, the use of the sensitive HemoCue HbAlc system may contribute to preventing complications in diabetics. Furthermore, treatment in the early stage of diabetes can improve the outcome of diabetes management and reduce the risk of severe complications [42].

In addition to its potential for screening, POCT HbAlc using the HemoCue system can be a suitable alternative to monitoring diabetic patients, as it is less costly, rapid, and only requires a small finger prick blood sample compared to the standard laboratory method (Table 1), providing an immediate result [30]. Also, the POCT-HbA1c tool with instant results and affordable price could be an alternative method to increase patient compliance with diabetic medicine, thereby maintaining their blood glucose level in the normal range to improve the outcome of diabetes management [18].

\section{Conclusions}

POCT-HbAlc measurements using the HemoCue HbAlc system, compared to the standard laboratory method, are highly sensitive and accurate, as well as being less timeconsuming and costly, with a more convenient blood collection. Therefore, this POCT system is a suitable tool for diabetes screening and management in the primary health care service, clinics, or remote areas with limited access to a laboratory. However, HbAlc measurement using the standard laboratory method is still recommended for the initial diabetes diagnosis.

\section{Data Availability}

The data supporting the findings of this study are available within the supplementary material.

\section{Additional Points}

Strengths and Limitations of This Study. (i) Our study shows that POCT using the HemoCue ${ }^{\circledR} \mathrm{HbA1c} 501$ system is sensitive but less specific compared to the standard laboratory diagnostic method. POCT-HbAlc makes it possible to monitor patient prognosis and compliance with the therapy regimen in one visit. (ii) Analysis of the agreement of the results from the two methods shows that the POCT-HbAlc can replace the more expensive and time-consuming standard lab method for use in the management of people with DM. (iii) This cross-sectional study had a limited number of participants.

\section{Conflicts of Interest}

The authors declare that there is no conflict of interest regarding the publication of this article.

\section{Authors' Contributions}

A. B. designed the study, contributed to the analysis, wrote the article, and gave final approval of the version to be submitted. R. R. designed the study and helped drafting the article. N. R. and A. I. C were responsible for data interpretation and writing the article and contributed to the analysis.

\section{Acknowledgments}

We thank Tenny Putri Wikayani and Nurul Qomarilla for their technical support and Kurnia Wahyudi for statistical analysis support. This work was funded by the Indonesian Science Fund (Dana Ilmu Pengetahuan Indonesia, DIPI) and Indonesian Endowment Fund for Education (Lembaga Pengelola Dana Pendidikan, LPDP), grant no. MR/P017568/1.

\section{Supplementary Materials}

Supplementary 1: comparison of HbA1c measured by diagnostic lab and POCT HemoCue. (Supplementary Materials)

\section{References}

[1] K. G. M. M. Alberti and P. Z. Zimmet, "Definition, diagnosis and classification of diabetes mellitus and its complications. Part 1: diagnosis and classification of diabetes mellitus. Provisional report of a WHO consultation," Diabetic Medicine, vol. 15, no. 7, pp. 539-553, 1998. 
[2] J.-P. Bastard, M. Maachi, C. Lagathu et al., "Recent advances in the relationship between obesity, inflammation, and insulin resistance," European Cytokine Network, vol. 17, no. 1, pp. 412,2006

[3] S. C. Larsson, N. Orsini, and A. Wolk, "Diabetes mellitus and risk of colorectal cancer: a meta-analysis," Journal of the National Cancer Institute, vol. 97, no. 22, pp. 1679-1687, 2005.

[4] M. E. Stegenga, S. N. van der Crabben, R. M. E. Blümer et al., "Hyperglycemia enhances coagulation and reduces neutrophil degranulation, whereas hyperinsulinemia inhibits fibrinolysis during human endotoxemia," Blood, vol. 112, no. 1, pp. 82$89,2008$.

[5] S. Pavlou, J. Lindsay, R. Ingram, H. Xu, and M. Chen, "Sustained high glucose exposure sensitizes macrophage responses to cytokine stimuli but reduces their phagocytic activity," $B M C$ Immunology, vol. 19, no. 1, p. 24, 2018.

[6] J. A. Suaya, D. F. Eisenberg, C. Fang, and L. G. Miller, "Skin and soft tissue infections and associated complications among commercially insured patients aged 0-64 years with and without diabetes in the U.S," PLoS One, vol. 8, no. 4, article e60057, 2013.

[7] M. Ferracini, J. O. Martins, M. R. M. Campos, D. B. C. Anger, and S. Jancar, "Impaired phagocytosis by alveolar macrophages from diabetic rats is related to the deficient coupling of LTs to the Fc $\gamma$ R signaling cascade," Molecular Immunology, vol. 47, no. 11-12, pp. 1974-1980, 2010.

[8] A. Berbudi, N. Rahmadika, A. I. Tjahjadi, and R. Ruslami, "Type 2 diabetes and its impact on the immune system," Current Diabetes Reviews, vol. 16, no. 5, pp. 442-449, 2020.

[9] Group UKPDPDS (UKPDS), "UK prospective diabetes study 16. Overview of 6 years' therapy of type II diabetes: a progressive disease," Diabetes, vol. 44, no. 11, pp. 1249-1258, 1995.

[10] Group UPDS (UKPDS), "Intensive blood-glucose control with sulphonylureas or insulin compared with conventional treatment and risk of complications in patients with type 2 diabetes (UKPDS 33)," The Lancet, vol. 352, no. 9131, pp. 837-853, 1998.

[11] Group TDC and CTR, "The effect of intensive treatment of diabetes on the development and progression of long-term complications in insulin-dependent diabetes mellitus," The New England Journal of Medicine, vol. 329, no. 14, pp. 977986, 1993.

[12] R. R. Little, "Glycated hemoglobin standardization - National Glycohemoglobin Standardization Program (NGSP) perspective," Clinical Chemistry and Laboratory Medicine, vol. 41, no. 9, pp. 1191-1198, 2003.

[13] R. R. Little and C. L. Rohlfing, "HbA1cstandardization: background, progress and current issues," Laboratory Medicine, vol. 40, no. 6, pp. 368-373, 2009.

[14] D. M. Nathan, H. Turgeon, and S. Regan, "Relationship between glycated haemoglobin levels and mean glucose levels over time," Diabetologia, vol. 50, no. 11, pp. 2239-2244, 2007.

[15] American Diabetes Association, "2. Classification and diagnosis of diabetes: standards of medical care in diabetesd-2018," Diabetes Care, vol. 41, pp. S13-S27, 2018.

[16] C. D. Miller, C. S. Barnes, L. S. Phillips et al., "Rapid A1c availability improves clinical decision-making in an urban primary care clinic," Diabetes Care, vol. 26, no. 4, pp. 11581163, 2003.

[17] E. Cagliero, E. V. Levina, and D. M. Nathan, "Immediate feedback of HbA1c levels improves glycemic control in type 1 and insulin-treated type 2 diabetic patients," Diabetes Care, vol. 22, no. 11, pp. 1785-1789, 1999.

[18] M. Heisler, J. D. Piette, M. Spencer, E. Kieffer, and S. Vijan, "The relationship between knowledge of recent $\mathrm{HbA1c}$ values and diabetes care understanding and self-management," Diabetes Care, vol. 28, no. 4, pp. 816-822, 2005.

[19] J.-H. Cho, S.-A. Chang, H.-S. Kwon et al., "Long-term effect of the internet-based glucose monitoring system on $\mathrm{HbAlc}$ reduction and glucose stability: a 30-month follow-up study for diabetes management with a ubiquitous medical care system," Diabetes Care, vol. 29, no. 12, pp. 2625-2631, 2006.

[20] American Diabetes Association, "Standards of medical care in diabetes-2010," Diabetes Care, vol. 33, Supplement 1, pp. S11S61, 2009.

[21] "Glycated haemoglobin (HbA1c) for the diagnosis of diabetes," 2011, December 2018, https://www.ncbi.nlm.nih.gov/ books/NBK304271/.

[22] W. G. John, "Use of HbA1c in the diagnosis of diabetes mellitus in the UK. The implementation of World Health Organization guidance 2011," Diabetic Medicine, vol. 29, no. 11, pp. 1350-1357, 2012.

[23] New Zealand Society for the Study of Diabetes, "NZSSD position statement on the diagnosis of, and screening for, type 2 diabetes," 2011, August 2019, https://www.nzssd.org.nz.

[24] J. Parsons, A. Vogan, J. Morona et al.et al., "HbAlc testing in the diagnosis of diabetes mellitu . AHTA 2014s," August 2019, http://www.msac.gov.au/http://www.msac.gov.au/http://www. msac.gov.au/.

[25] M. C. D’Emden, J. E. Shaw, P. G. Colman et al., "The role of HbAlc in the diagnosis of diabetes mellitus in Australia," The Medical Journal of Australia, vol. 197, no. 4, pp. 220-221, 2012.

[26] C. Elley, T. Kenealy, E. Robinson, and P. L. Drury, “Glycated haemoglobin and cardiovascular outcomes in people with type 2 diabetes: a large prospective cohort study," Diabetic Medicine, vol. 25, pp. 1295-1301, 2008.

[27] E. Selvin, S. Marinopoulos, G. Berkenblit et al., "Meta-analysis: glycosylated hemoglobin and cardiovascular disease in diabetes mellitus," Annals of Internal Medicine, vol. 141, no. 6, pp. 421-431, 2004.

[28] A. Rudijanto, P. Soewondo, S. Waspadji, E. Yunir, and D. Purnamasari, "The Indonesian Society of Endocrinology's summary article of diabetes mellitus national clinical practice guidelines," Journal of the ASEAN Federation of Endocrine Societies, vol. 26, no. 1, pp. 17-19, 2011.

[29] P. Soewondo, A. Ferrario, and D. Tahapary, "Challenges in diabetes management in Indonesia: a literature review," Globalization and Health, vol. 9, no. 1, p. 63, 2013.

[30] C. P. Price, "Point of care testing," BMJ, vol. 322, no. 7297, pp. 1285-1288, 2001.

[31] J. V. Marley, M. S. Oh, N. T. Hadgraft, S. L. Singleton, K. Isaacs, and D. N. Atkinson, "Using glycated haemoglobin testing to simplify diabetes screening in remote aboriginal Australian health care settings," The Medical Journal of Australia, vol. 203, no. 1, pp. 28-32, 2015.

[32] J. Knaebel, B. R. Irvin, and C. Z. Xie, "Accuracy and clinical utility of a point-of-care $\mathrm{HbA}_{1 \mathrm{c}}$, testing device," Postgraduate Medicine, vol. 125, pp. 91-98, 2015.

[33] E. Lenters-Westra and R. J. Slingerland, "Three of 7 hemoglobin A1c point-of-care instruments do not meet generally accepted analytical performance criteria," Clinical Chemistry, vol. 60, no. 8, pp. 1062-1072, 2014. 
[34] P. Chamnan, R. K. Simmons, N. G. Forouhi et al., "Incidence of type 2 diabetes using proposed $\mathrm{HbAlc}$ diagnostic criteria in the European prospective investigation of cancer-Norfolk cohort: implications for preventive strategies," Diabetes Care, vol. 34, no. 4, pp. 950-956, 2011.

[35] Y. Handelsman, J. I. Mechanick, L. Blonde et al., "American Association of Clinical Endocrinologists Medical Guidelines for Clinical Practice for developing a diabetes mellitus comprehensive care plan," Endocrine Practice, vol. 17, Supplement 2, pp. 1-53, 2011.

[36] R. Grant, A. S. Adams, C. M. Trinacty et al., "Relationship between patient medication adherence and subsequent clinical inertia in type 2 diabetes glycemic management," Diabetes Care, vol. 30, no. 4, pp. 807-812, 2007.

[37] K. K. Hood, C. M. Peterson, J. M. Rohan, and D. Drotar, "Association between adherence and glycemic control in pediatric type 1 diabetes: a meta-analysis," Pediatrics, vol. 124, no. 6, pp. e1171-e1179, 2009.

[38] E. Lenters-Westra and R. J. Slingerland, "Six of eight hemoglobin $A_{1 c}$ point-of-care instruments do not meet the general accepted analytical performance criteria," Clinical Chemistry, vol. 56, no. 1, pp. 44-52, 2010.

[39] D. E. Bruns and J. C. Boyd, "Few point-of-care hemoglobin A1c assay methods meet clinical needs," Clinical Chemistry, vol. 56, no. 1, pp. 4-6, 2010.

[40] K. Khunti, M. A. Stone, A. C. Burden et al., "Randomised controlled trial of near-patient testing for glycated haemoglobin in people with type 2 diabetes mellitus," The British Journal of General Practice, vol. 56, no. 528, pp. 511-517, 2006.

[41] C. Reily, T. J. Stewart, M. B. Renfrow, and J. Novak, "Glycosylation in health and disease," Nature Reviews Nephrology, vol. 15, no. 6, pp. 346-366, 2019.

[42] W. H. Herman, W. Ye, S. J. Griffin et al., "Early detection and treatment of type 2 diabetes reduce cardiovascular morbidity and mortality: a simulation of the results of the AngloDanish-Dutch study of intensive treatment in people with screen-detected diabetes in primary care (ADDITIONEurope)," Diabetes Care, vol. 38, no. 8, pp. 1449-1455, 2015. 